Rev. Mat. Iberoamericana 19 (2003), 221-234

\title{
Mapping properties of the elliptic maximal function
}

\section{Burak Erdoğan}

\begin{abstract}
We prove that the elliptic maximal function maps the Sobolev space $W_{4, \eta}\left(\mathbb{R}^{2}\right)$ into $L^{4}\left(\mathbb{R}^{2}\right)$ for all $\eta>1 / 6$. The main ingredients of the proof are an analysis of the intersection properties of elliptic annuli and a combinatorial method of Kolasa and Wolff.
\end{abstract}

\section{Introduction}

In 1986, Bourgain [1] proved that the circular maximal function

$$
M_{C} f(x)=\sup _{t>0} \int_{S^{1}} f(x+t s) d \sigma(s)
$$

is bounded on $L^{p}\left(\mathbb{R}^{2}\right)$ if $p>2$. Different proofs were given in [7] and [10]. In [8], Schlag generalized this result and obtained almost sharp $L^{p} \rightarrow L^{q}$ estimates for $M_{C}$.

In this paper, we attempt to generalize Bourgain's theorem in a different direction; we consider a natural generalization of the circular maximal function by taking maximal averages over ellipses instead of circles.

More explicitly, let $\mathcal{E}$ be the set of all ellipses in $\mathbb{R}^{2}$ centered at the origin with axial lengths in $\left[\frac{1}{2}, 2\right]$. Note that we do not restrict ourselves to the ellipses whose axes are parallel to the co-ordinate axes. The elliptic maximal function, $M$, is defined in the following way: Let $f$ be a real-valued continuous function on $\mathbb{R}^{2}$, then

$$
M f(x)=\sup _{E \in \mathcal{E}} \frac{1}{|E|} \int_{E} f(x+s) d \sigma(s), \quad x \in \mathbb{R}^{2},
$$

where $d \sigma$ is the arclength measure on $E$ and $|E|$ is the length of $E$.

2000 Mathematics Subject Classification: Primary 42B25.

Keywords: Multiparameter maximal functions, circular maximal function, Sobolev space estimates. 
We are interested in the $L^{p}$ mapping properties of $M$.

Proposition $1 M$ is not bounded in $L^{p}$ for $p \leq 4$.

Proof. First, we prove that $M$ is not bounded in $L^{p}$ for $p<4$. Let $f_{\delta}$ be the characteristic function of the $\delta$-neighborhood of the unit circle. A simple calculation shows that for all $x \in B(0,1), M f_{\delta}(x) \gtrsim \delta^{1 / 4}$. This is because of the fact that for all $x \in B(0,1)$, there is an ellipse centered at $x$ which is third order tangent to the unit circle. Therefore, $\left\|M f_{\delta}\right\|_{p} \gtrsim \delta^{1 / 4}$, whereas $\left\|f_{\delta}\right\|_{p} \approx \delta^{1 / p}$. Taking the limit $\delta \rightarrow 0$ yields the claim.

To prove that $M$ is not bounded in $L^{4}$, consider the function

$$
g_{\delta}(x)=(|1-| x||+\delta)^{-1 / 4} \chi_{B(0,2) \backslash B(0,1)} .
$$

Note that $\left\|g_{\delta}\right\|_{4} \approx \log (1 / \delta)^{1 / 4}$. On the other hand, we have $M g_{\delta}(x) \gtrsim$ $\log (1 / \delta)$ for all $x \in B(0,1)$ and hence $\left\|M g_{\delta}\right\|_{4} \gtrsim \log (1 / \delta)$ (see [8] for the details).

In light of Proposition 1, one may conjecture that $M$ is bounded in $L^{p}$ for $p>4$. We are far from proving this conjecture. However, we obtain some estimates for $M$ in this direction. We state our results for the key exponent $p=4$.

The setup is the following; we work with the family of maximal functions:

$$
M_{\delta} f(x)=\sup _{E \in \mathcal{E}} \frac{1}{\left|E^{\delta}\right|} \int_{x+E^{\delta}} f(u) d u
$$

where $E^{\delta}$ is the $\delta$ neighborhood of the ellipse $E$ and $\left|E^{\delta}\right|$ is the two-dimensional Lebesgue measure of $E^{\delta}$. We investigate the $L^{4}$ mapping properties of $M_{\delta}$.

Applying $M_{\delta}$ to the functions in (1.2), we see that the inequality

$$
\left\|M_{\delta} f\right\|_{4} \lesssim A(\delta)\|f\|_{4}, \quad \delta>0
$$

can not hold if $A(\delta)=o\left(\log (1 / \delta)^{3 / 4}\right)$. On the other hand, estimating the right-hand side of (1.3) by $\delta^{-1}\|f\|_{1}$ implies that $\left\|M_{\delta} f\right\|_{1} \lesssim \delta^{-1}\|f\|_{1}$ and estimating it by $\|f\|_{\infty}$ implies that $\left\|M_{\delta} f\right\|_{\infty} \leq\|f\|_{\infty}$. By interpolating these bounds, we see that (1.4) holds for $A(\delta) \gtrsim \delta^{-1 / 4}$.

Let $E^{\delta}$ denote the $\delta$-neighborhood of the ellipse $E$. We have the following basic property of the elliptic annuli. It corresponds to the fact that two distinct ellipses can be at most third order tangent to each other. 
Lemma 2 Let $E_{1}$ and $E_{2}$ be ellipses such that the distance $\Delta$ between their centers is $\gtrsim \delta^{2 / 5}$. Then

$$
\left|E_{1}^{\delta} \cap E_{2}^{\delta}\right| \lesssim \frac{\delta^{5 / 4}}{\Delta^{1 / 4}}
$$

We prove this lemma in Section 3 (Corollary 10 (i)). Now, using this lemma and Cordoba's $L^{2}$ Kakeya argument [2], we prove the simple fact that (1.4) holds for $A(\delta) \gtrsim \delta^{-3 / 16}$.

\section{Lemma 3}

$$
\left\|M_{\delta} f\right\|_{4} \lesssim \delta^{-3 / 16}\|f\|_{4}, \quad \delta>0
$$

Proof. The lemma follows by interpolating the trivial $L^{\infty}$ bound with the following restricted weak type estimate:

$$
\left\|M_{\delta} f\right\|_{2, \infty} \lesssim \delta^{-3 / 8}\|f\|_{2,1} .
$$

Fix a set $A$ in $B(0,1)$ and $\lambda \in[0,1]$. Let $\Omega=\left\{x: M_{\delta}\left(\chi_{A}\right)>\lambda\right\}$. Take a $\delta$-separated set $\left\{x_{1}, \ldots, x_{m}\right\}$ in $\Omega$. We have

$$
|\Omega| \lesssim m \delta^{2}
$$

For each $x_{j}$, choose an ellipse $E_{j}$ such that $\left|E_{j}^{\delta} \cap A\right|>\lambda \delta$. Using CauchySchwarz inequality, we have

$$
\begin{aligned}
m \delta \lambda & \leq \sum_{j=1}^{m}\left|E_{j}^{\delta} \cap A\right|=\int_{A} \sum_{j} \chi_{E_{j}^{\delta}} \\
& \leq|A|^{1 / 2}\left\|\sum_{j} \chi_{E_{j}^{\delta}}\right\|_{2} \\
& =|A|^{1 / 2}\left(\sum_{j, k}\left|E_{j}^{\delta} \cap E_{k}^{\delta}\right|\right)^{1 / 2} .
\end{aligned}
$$

Now, we estimate the sum $\sum_{j, k}\left|E_{j}^{\delta} \cap E_{k}^{\delta}\right|$ using Lemma 2. We have

$$
\left|E_{j}^{\delta} \cap E_{k}^{\delta}\right| \lesssim \frac{\delta^{5 / 4}}{\left|x_{j}-x_{k}\right|^{1 / 4}}
$$

given that $\left|x_{j}-x_{k}\right| \gtrsim \delta^{2 / 5}$. Using this, we obtain for fixed $j$

$$
\begin{aligned}
\sum_{k}\left|E_{j}^{\delta} \cap E_{k}^{\delta}\right| & \lesssim \delta^{-2} \int_{1 \gtrsim\left|x_{j}-x\right| \gtrsim \delta^{2 / 5}} \frac{\delta^{5 / 4}}{\left|x_{j}-x\right|^{1 / 4}} d x+\delta^{-2} \int_{\left|x_{j}-x\right| \delta^{2 / 5}} \delta d x \\
& \lesssim \delta^{-3 / 4} .
\end{aligned}
$$


Thus,

$$
\sum_{j, k}\left|E_{j}^{\delta} \cap E_{k}^{\delta}\right| \lesssim m \delta^{-3 / 4}
$$

Using (1.9) in (1.7), we have

$$
m \delta \lambda \lesssim|A|^{1 / 2}\left(m \delta^{-3 / 4}\right)^{1 / 2}
$$

Hence

$$
|\Omega| \lesssim m \delta^{2} \lesssim\left(\delta^{-3 / 8} \frac{|A|^{1 / 2}}{\lambda}\right)^{2}
$$

which proves (1.5).

We have the following improvement:

Theorem 4 For all $\varepsilon>0$, inequality (1.4) holds with $A(\delta)=\delta^{-1 / 6} \delta^{-\varepsilon}$.

Remark. Theorem 4 implies that $M$ maps $W_{4,1 / 6+\varepsilon}$ into $L^{4}$ for all $\varepsilon>0$. Here $W_{p, \eta}$ is the Sobolev space consisting of functions $f$ such that

$$
\left\|(1-\Delta)^{\eta / 2} f\right\|_{p}<\infty .
$$

Theorem 4 is a corollary of the following stronger theorem, which is the main result of this paper.

Theorem $5\left\|M_{\delta} f\right\|_{24 / 7, \infty} \lesssim \delta^{-1 / 3}|\log (\delta)|^{5 / 4}\|f\|_{2,1}$.

Proof of Theorem 5 utilizes an analysis of the intersection properties of elliptic annuli. Lemma 2 above and the following lemma are the basic elements of the proof; we prove them in section 3. The following lemma can be considered as a Marstrand's three circle lemma type result for ellipses.

Lemma 6 Fix $\Delta \gtrsim \delta^{2 / 5}, d \gtrsim \delta$ and $u \gtrsim 1$. Take any two ellipses $E_{1}$ and $E_{2}$ such that the distance between their centers $\left(c_{1}, c_{2}\right.$ respectively) is approximately $d$. Then the $\delta$-entropy of the set

$S:=\left\{x \in \mathbb{R}^{2}:\left|x-c_{i}\right| \gtrsim \Delta, i=1,2, \exists\right.$ an ellipse $E$ centered at $x$ such that $\left.\left|E^{\delta} \cap E_{i}^{\delta}\right| \gtrsim \delta(\delta / u \Delta)^{1 / 4}, i=1,2,\right\}$

$i s \lesssim \frac{1}{\delta^{2} d^{1 / 2}}|\log (\delta)| u^{3 / 4}(\delta / \Delta)^{1 / 4}$ 
Note that in the proof of Lemma 3 (inequality (1.8)), we assumed that any two ellipses can be third order tangent to each other in a given set of ellipses. However, using Lemma 6 and a combinatorial method of Kolasa and Wolff [4], [11], we can bound the number of pairs of elliptic annuli which are third order tangent to each other. This is the main ingredient of the proof of Theorem 5 .

This technique was also used in [8], [10], [9] and [6].

\section{Notation:}

$S^{1}$ : the unit circle.

$E_{z}^{e, f}:=\left\{x \in \mathbb{R}^{2}:\left(\frac{x_{1}-z_{1}}{e}\right)^{2}+\left(\frac{x_{2}-z_{2}}{f}\right)^{2}=1\right\}$.

$E^{\delta}: \delta$ neighborhood of the ellipse $E$.

$K$ : A constant which may vary from line to line.

$A \lesssim B: A \leq K B$

$A \approx B: A \lesssim B$ and $A \gtrsim B$.

$A \ll B: A \leq K^{-1} B$ where $K$ is a large enough constant.

$|A|$ : cardinality or the measure of the set $A$ or the length of the vector $A$ in $\mathbb{R}^{2}$.

\section{Proof of Theorem 5}

Let $A \subset \mathbb{R}^{2}, 0<\lambda \leq 1$ and $\Omega=\left\{x \in \mathbb{R}^{2}: M_{\delta} \chi_{A}(x)>\lambda\right\}$. We need to prove that

$$
|\Omega| \lesssim\left(|\log (\delta)|^{5 / 4} \delta^{-1 / 3} \frac{|A|^{1 / 2}}{\lambda}\right)^{24 / 7},
$$

Without loss of generality, we can assume that $A \subset D(0,1)$. Let $\left\{x_{j}\right\}_{j=1}^{m}$ be a maximally $\delta$ separated set in $\Omega$. Note that

$$
|\Omega| \lesssim m \delta^{2}
$$

Choose ellipses $E_{j}$ centered at $x_{j}$ such that $\left|E_{j}^{\delta} \cap A\right|>\lambda\left|E_{j}^{\delta}\right| \approx \lambda \delta$. We have

$$
\begin{aligned}
m \delta \lambda & \lesssim \sum_{j=1}^{m}\left|E_{j}^{\delta} \cap A\right|=\int_{A} \sum_{j=1}^{m} \chi_{E_{j}^{\delta}} \\
& \leq|A|^{1 / 2}\left\|\sum_{j=1}^{m} \chi_{E_{j}^{\delta}}\right\|_{2}=|A|^{1 / 2}\left(\sum_{j, k=1}^{m}\left|E_{j}^{\delta} \cap E_{k}^{\delta}\right|\right)^{1 / 2}
\end{aligned}
$$


Let

$$
S_{\Delta, u}=\left\{(j, k):\left|x_{j}-x_{k}\right| \in(\Delta, 2 \Delta), \delta\left(\frac{\delta}{u \Delta}\right)^{1 / 4} \leq\left|E_{j}^{\delta} \cap E_{k}^{\delta}\right| \leq \delta\left(\frac{\delta}{2 u \Delta}\right)^{1 / 4}\right\} .
$$

Using this notation, we can estimate $\sum_{j, k=1}^{m}\left|E_{j}^{\delta} \cap E_{k}^{\delta}\right|$ as

$$
\begin{aligned}
\sum_{j, k=1}^{m}\left|E_{j}^{\delta} \cap E_{k}^{\delta}\right| & \lesssim \sum_{\delta^{2 / 5} \lesssim \Delta \lesssim 1} \sum_{u}\left|S_{\Delta, u}\right| \delta\left(\frac{\delta}{u \Delta}\right)^{1 / 4}+\sum_{j=1}^{m} \delta \min \left(m, \delta^{-6 / 5}\right) \\
& \lesssim \sum_{\delta^{2 / 5} \lesssim \Delta \lesssim 1} \sum_{u}\left|S_{\Delta, u}\right| \delta\left(\frac{\delta}{u \Delta}\right)^{1 / 4}+m^{17 / 12} \delta^{3 / 10}
\end{aligned}
$$

where the summations are over the dyadic values of $\Delta$ and the dyadic values of $u \in\left(1, \delta^{-K}\right)$ (since the terms with $u$ greater than a high power of $\delta^{-1}$ makes negligible contribution, and Lemma 2 implies that $S_{\Delta, u}$ is empty if $\Delta>\delta^{2 / 5}$ and $\left.u \ll 1\right)$.

Now, we find a bound for the cardinality of the set $S_{\Delta, u}$ using Lemma 6 . Consider the set of triples:

$$
\begin{aligned}
Q=\left\{\left(j, k_{1}, k_{2}\right):\right. & \left|x_{j}-x_{k_{i}}\right| \in(\Delta, 2 \Delta), \\
& \left.\delta\left(\frac{\delta}{u \Delta}\right)^{1 / 4} \leq\left|E_{j}^{\delta} \cap E_{k_{i}}^{\delta}\right| \leq \delta\left(\frac{\delta}{2 u \Delta}\right)^{1 / 4}, i=1,2\right\} .
\end{aligned}
$$

We calculate the cardinality of $Q$ in two different ways. Let $S_{j}=\mid\{k$ : $\left.(j, k) \in S_{\Delta, u}\right\} \mid$. Note that there are at least $S_{j}^{2}$ triples in $Q$ whose first co-ordinate is $j$. Hence, we have

$$
\left|S_{\Delta, u}\right|=\sum_{j=1}^{m} S_{j} \leq m^{1 / 2}\left(\sum_{j=1}^{m} S_{j}^{2}\right)^{1 / 2} \lesssim(m|Q|)^{1 / 2} .
$$

On the other hand, we can choose $k_{1}$ in $m$ different ways, and for fixed $k_{1}$, there are at most $\min \left(m, \frac{d^{2}}{\delta^{2}}\right)$ indices $k_{2}$ such that $\left|x_{k_{2}}-x_{k_{1}}\right| \in(d, 2 d)$. For any such $\left(k_{1}, k_{2}\right)$, by Lemma 6 and $\delta$-separatedness, there are at most $\frac{1}{\delta^{2}} \min \left(|\log (\delta)| d^{-1 / 2} u^{3 / 4}(\delta / \Delta)^{1 / 4}, \Delta^{2}\right)$ indices $j$ such that $\left(j, k_{1}, k_{2}\right) \in Q$. Summing over dyadic $d \in(\delta, 1)$, we obtain

$$
\begin{aligned}
|Q| & \lesssim m \sum_{d} \min \left(m, \frac{d^{2}}{\delta^{2}}\right) \frac{1}{\delta^{2}} \min \left(|\log (\delta)| d^{-1 / 2} u^{3 / 4}(\delta / \Delta)^{1 / 4}, \Delta^{2}\right) \\
& \lesssim \frac{m}{\delta^{2}}|\log (\delta)| \min \left(\frac{(m u)^{3 / 4}}{(\delta \Delta)^{1 / 4}}, m \Delta^{2}\right) .
\end{aligned}
$$


Using (2.5) in (2.4), we have

$$
\begin{aligned}
\left|S_{\Delta, u}\right| & \lesssim(m|Q|)^{1 / 2} \lesssim \frac{m}{\delta}|\log (\delta)|^{1 / 2} \min \left(\frac{(m u)^{3 / 8}}{(\delta \Delta)^{1 / 8}}, m^{1 / 2} \Delta\right) \\
& \lesssim \frac{m}{\delta}|\log (\delta)|^{1 / 2}\left(\frac{(m u)^{3 / 8}}{(\delta \Delta)^{1 / 8}}\right)^{2 / 3}\left(m^{1 / 2} \Delta\right)^{1 / 3} \\
& \lesssim \frac{m^{17 / 12}}{\delta^{13 / 12}}|\log (\delta)|^{1 / 2}(u \Delta)^{1 / 4} .
\end{aligned}
$$

Using (2.6) in (2.3) together with the fact that there are at most $\log (\delta)^{2}$ terms in the summation, we obtain

$$
\begin{aligned}
\sum_{j, k=1}^{m}\left|E_{j}^{\delta} \cap E_{k}^{\delta}\right| & \lesssim \sum_{\Delta} \sum_{u} \delta\left(\frac{\delta}{u \Delta}\right)^{1 / 4} \frac{m^{17 / 12}}{\delta^{13 / 12}}|\log (\delta)|^{1 / 2}(u \Delta)^{1 / 4} \\
& +m^{17 / 12} \delta^{3 / 10} \\
\lesssim & m^{17 / 12} \delta^{1 / 6}|\log (\delta)|^{5 / 2}
\end{aligned}
$$

Using (2.7), (2.2) and (2.1), we have

$$
|\Omega| \lesssim m \delta^{2} \lesssim\left(|\log (\delta)|^{5 / 4} \delta^{-1 / 3} \frac{|A|^{1 / 2}}{\lambda}\right)^{24 / 7}
$$

which yields the claim of the theorem.

\section{Proof of Lemmas 2 and 6}

Let $\mathcal{N}(A, \delta)$ denote the $\delta$ neighborhood of the set $A$. First, we find a relationship between the parameters $z_{1}, z_{2}, e$ and $f$ of an ellipse $E_{z}^{e, f}$ and the measure of the set $\mathcal{N}\left(E_{z}^{e, f}, \delta\right) \cap \mathcal{N}\left(S^{1}, \delta\right)$. We begin with the following basic lemma.

Lemma 7 Let $N$ be a positive integer. There exist constants $K_{1}$ and $K_{2}$ such that for all $\alpha>0$ and for all $\delta>0$, we have

$$
\begin{aligned}
\sum_{i=0}^{N}\left|a_{i}\right| \alpha^{i}>\delta \Longrightarrow & \exists x_{1} \in\left(0, K_{1} \alpha\right) \text { and } x_{2} \in\left(-K_{1} \alpha, 0\right) \\
& \text { such that }\left|\sum_{i=0}^{N} a_{i} x_{j}^{i}\right|>K_{2} \delta, \quad j=1,2 .
\end{aligned}
$$

Proof. The statement is trivial if $\alpha=1$, and the general case follows from this by the change of variable $y=x \alpha$. 
Let $S_{1}^{1}$ be $S^{1} \cap\left\{x \in \mathbb{R}^{2}: x_{2}>0,\left|x_{1}\right|<2 / 3\right\}$, and $d(x, y)$ denotes the distance between the points $x, y \in \mathbb{R}^{2}$.

Theorem 8 Let $d(z, 0)=\Delta \gtrsim \delta^{2 / 5}$. Then

i) The arclength of $E_{z}^{e, f} \cap \mathcal{N}\left(S_{1}^{1}, \delta\right)$ is $\lesssim\left(\frac{\delta}{\Delta}\right)^{1 / 4}$.

ii) If the arclength of the intersection is $\gtrsim\left(\frac{\delta}{u \Delta}\right)^{1 / 4}$ for some $1 \lesssim u \ll$ $(\Delta / \delta)^{1 / 3}$, then we have

$$
\begin{aligned}
\left|z_{1}\right| & \lesssim \min \left(u^{3 / 2}(\delta \Delta)^{1 / 2}, u^{9 / 4}(\delta / \Delta)^{3 / 4}\right) \\
\left|f-e^{2}\right| & \lesssim \min \left((u \Delta)^{3 / 4} \delta^{1 / 4}, u^{3 / 2}(\delta / \Delta)^{1 / 2}\right) \\
\left|z_{2}+f-1\right| & \lesssim \min \left((u \Delta)^{3 / 4} \delta^{1 / 4}, u^{3 / 2}(\delta / \Delta)^{1 / 2}\right)
\end{aligned}
$$

Proof. Consider the function

$$
f(x):=z_{2}+f\left(1-\left(\left(x-z_{1}\right) / e\right)^{2}\right)^{1 / 2}-\left(1-x^{2}\right)^{1 / 2} .
$$

Take a point $t \in(-2 / 3,2 / 3)$ such that $|f(t)|<\delta$. Note that the set $E_{z}^{e, f} \cap$ $\mathcal{N}\left(S^{1}, \delta\right)$ consists of at most four connected components. Hence, it suffices to prove that there exists $x_{1} \in\left(t-\left(\frac{\delta}{\Delta}\right)^{1 / 4}, t\right)$ and $x_{2} \in\left(t, t+\left(\frac{\delta}{\Delta}\right)^{1 / 4}\right)$ such that $\left|f\left(x_{j}\right)\right|>\delta$ for $j=1,2$, and if $x_{1}$ or $x_{2}$ are not in the $\left(\frac{\delta}{u \Delta}\right)^{1 / 4}$ neighborhood of $t$ for $1 \lesssim u \ll(\Delta / \delta)^{1 / 3}$, then (3.1), (3.2) and (3.3) are valid.

We consider the first five terms of the Taylor expansion of $f(x)$ around $t$. Let $w:=\left(1-\left(t-z_{1}\right)^{2} / e^{2}\right)^{-1 / 2}\left(1-t^{2}\right)^{1 / 2}$. We can assume that $w \approx 1$.

$$
\begin{aligned}
f(x)= & z_{2}+\left(f w^{-1}-1\right)\left(1-t^{2}\right)^{1 / 2} \\
& +\left[\left(\frac{f}{e^{2}}\left(z_{1}-t\right) w+t\right)\left(1-t^{2}\right)^{-1 / 2}\right](x-t) \\
+ & \frac{1}{2}\left[\left(1-t^{2}\right)^{-3 / 2}\left(1-\frac{f}{e^{2}} w^{3}\right)\right](x-t)^{2} \\
+ & \frac{1}{2}\left[\left(1-t^{2}\right)^{-5 / 2}\left(t-w^{5} \frac{f}{e^{4}}\left(t-z_{1}\right)\right)\right](x-t)^{3} \\
+ & \frac{1}{8}\left[\left(1-t^{2}\right)^{-7 / 2}\left(1+4 t^{2}-w^{7} \frac{f}{e^{4}}\left(1+4\left(t-z_{1}\right)^{2} / e^{2}\right)\right)\right](x-t)^{4} \\
& +\frac{1}{24}\left[\eta \frac{3+4 \eta^{2}}{\left(1-\eta^{2}\right)^{9 / 2}}-\frac{f}{e^{8}}\left(\eta-x_{1}\right) \frac{3 e^{2}+4\left(\eta-z_{1}\right)^{2}}{\left(1-\left(\eta-z_{1}\right)^{2} / e^{2}\right)^{9 / 2}}\right](\eta-t)^{5}, \\
=: & a_{0}+a_{1}(x-t)+a_{2}(x-t)^{2}+a_{3}(x-t)^{3}+a_{4}(x-t)^{4}+E r
\end{aligned}
$$


Choose $u$ such that $\sum_{i=0}^{4}\left|a_{i}\right|\left(\frac{\delta}{u \Delta}\right)^{i / 4}=\delta$, we have $\left|a_{i}\right| \leq(u \Delta)^{i / 4} \delta^{1-i / 4}$ for $i=0,1,2,3,4$. We have two cases:

(i) $u \gtrsim(\Delta / \delta)^{1 / 3}$. Lemma 7 shows that if we omit the error term $E r$, then the arclength of the intersection is $\lesssim\left(\frac{\delta}{\Delta}\right)^{1 / 3}$. It is easy to see using the hypothesis $\Delta \gtrsim \delta^{2 / 5}$ that the error term is not significant.

(ii) $u \ll(\Delta / \delta)^{1 / 3}$. Using the definitions of $a_{0}, a_{1}, a_{2}$ and $a_{3}$, we obtain

$$
\begin{aligned}
z_{2}\left(1-t^{2}\right)^{-1 / 2}+f w^{-1} & =1+O(\delta), \\
\frac{f}{e^{2}}\left(t-z_{1}\right) w & =t+O\left((u \Delta)^{1 / 4} \delta^{3 / 4}\right), \\
\frac{f}{e^{2}} w^{3} & =1+O\left((u \Delta)^{1 / 2} \delta^{1 / 2}\right), \\
\frac{f}{e^{4}}\left(t-z_{1}\right) w^{5} & =t+O\left((u \Delta)^{3 / 4} \delta^{1 / 4}\right) .
\end{aligned}
$$

Substituting (3.7) into (3.8), we obtain

$$
(e f)^{-2 / 3}\left(t-z_{1}\right)\left(1+O\left((u \Delta)^{1 / 2} \delta^{1 / 2}\right)\right)=t+O\left((u \Delta)^{3 / 4} \delta^{1 / 4}\right),
$$

which implies that

$$
\left(\frac{e^{1 / 3}}{f^{2 / 3}}-1\right) \frac{t-z_{1}}{e}+\frac{t-z_{1}}{e}-t=O\left((u \Delta)^{3 / 4} \delta^{1 / 4}\right) .
$$

Substituting (3.7) into (3.6), we obtain

$$
\frac{f^{2 / 3}}{e^{4 / 3}}\left(t-z_{1}\right)\left(1+O\left((u \Delta)^{1 / 2} \delta^{1 / 2}\right)\right)=t+O\left((u \Delta)^{1 / 4} \delta^{3 / 4}\right)
$$

which implies that

$$
t\left(1-\frac{e^{4 / 3}}{f^{2 / 3}}\right)=z_{1}+O\left(\left|z_{1}-t\right|(u \Delta)^{1 / 2} \delta^{1 / 2}+(u \Delta)^{1 / 4} \delta^{3 / 4}\right)
$$

Subtracting (3.9) from (3.11), we obtain

$$
\left(t-z_{1}\right)\left(f^{4 / 3}-e^{2 / 3}+O\left((u \Delta)^{1 / 2} \delta^{1 / 2}\right)\right)=O\left((u \Delta)^{3 / 4} \delta^{1 / 4}\right) .
$$

Substituting (3.7) into (3.5), we obtain

$$
z_{2}\left(1-t^{2}\right)^{-1 / 2}+\left(\frac{f^{4 / 3}}{e^{2 / 3}}-1\right)=O\left((u \Delta)^{1 / 2} \delta^{1 / 2}\right) .
$$


Now, there are two cases $\left|z_{2}\right| \approx \Delta$ or $\left|z_{1}\right| \approx \Delta$.

Case a) Assume $\left|z_{2}\right| \approx \Delta$. (3.14) implies that $\left|e-f^{2}\right| \approx \Delta$. Using this in (3.13), we obtain

$$
t-z_{1}=O\left(u^{3 / 4}(\delta / \Delta)^{1 / 4}\right),
$$

which implies using (3.11) that

$$
t=O\left(u^{3 / 4}(\delta / \Delta)^{1 / 4}\right) .
$$

Using the fact $\left|e-f^{2}\right| \approx \Delta$ and (3.15) in (3.10), we obtain

$$
\frac{t-z_{1}}{e}-t=O\left((u \Delta)^{3 / 4} \delta^{1 / 4}\right) \text {. }
$$

This and the definition of $w$ implies that $w=1+O\left((u \Delta)^{3 / 4} \delta^{1 / 4}\right)$. On the other hand, using (3.15) and (3.16) in the definition of $w$, we obtain $w=1+O\left(u^{3 / 2}(\delta / \Delta)^{1 / 2}\right)$. Hence, using (3.7), we have

$$
\left|f-e^{2}\right| \lesssim \min \left((u \Delta)^{3 / 4} \delta^{1 / 4}, u^{3 / 2}(\delta / \Delta)^{1 / 2}\right) .
$$

Using (3.15), (3.16) and (3.17) in (3.12), we obtain

$$
\left|z_{1}\right| \lesssim \min \left(u^{3 / 2}(\delta \Delta)^{1 / 2}, u^{9 / 4}(\delta / \Delta)^{3 / 4}\right) .
$$

Finally, using (3.16) and the estimates for $|w-1|$ in (3.5), we obtain

$$
\left|z_{2}+f-1\right| \lesssim \min \left((u \Delta)^{3 / 4} \delta^{1 / 4}, u^{3 / 2}(\delta / \Delta)^{1 / 2}\right) .
$$

Case b) Assume $\left|z_{1}\right| \approx \Delta$. Using (3.12), we obtain

$$
\left|f-e^{2}\right| \approx \Delta,|t| \approx \Delta \text {. }
$$

Using (3.7), we obtain $\left(w^{2}-1\right)\left(f / e^{2}\right)^{2 / 3}+\left(f / e^{2}\right)^{2 / 3}-1=O\left((u \Delta)^{1 / 2} \delta^{1 / 2}\right)$, which implies using (3.20) that

$$
\left|w^{2}-1\right| \approx \Delta .
$$

Using the definition of $w$, we obtain $w^{2}-1 \approx\left(t-z_{1}\right)^{2} / e^{2}-t^{2}$. Hence (3.21) implies that

$$
\left|\frac{t-z_{1}}{e}-t\right| \approx \Delta
$$

Using (3.9), we obtain

$$
\left(\frac{e^{1 / 3}}{f^{2 / 3}}-1\right) \frac{t-z_{1}}{e}+\frac{t-z_{1}}{e}=t+O\left((u \Delta)^{3 / 4} \delta^{1 / 4}\right),
$$


which implies using (3.22) that

$$
\left|e-f^{2}\right|\left|t-z_{1}\right| \approx \Delta
$$

Hence $\left|e-f^{2}\right| \gtrsim \Delta$ and (3.14) implies that $\left|z_{2}\right| \gtrsim \Delta$. Thus the estimates that we obtained in case a) are valid.

Applying Lemma 7 (with $K_{1} \delta$ instead of the $\delta$ in the lemma, for a sufficiently large $\left.K_{1}\right)$, we see that $|f(x)-E r|>K \delta$, for some $z_{1} \in(t-$ $\left.K(\delta /(u \Delta))^{1 / 4}, 0\right)$ and $x_{2} \in\left(0, t+K(\delta /(u \Delta))^{1 / 4}\right)$.

Now, we prove that

$$
\operatorname{Er}=O(\delta)
$$

for $x \in\left(t-K(\delta /(u \Delta))^{1 / 4}, t+K(\delta /(u \Delta))^{1 / 4}\right)$. Note that the estimates that we obtained in part a) imply that $|e-1|,|f-1| \lesssim \Delta$. Let $h(\eta)=\eta \frac{3+4 \eta^{3}}{(1-\eta)^{9 / 2}}$. We have

$$
\begin{aligned}
|E r| & \lesssim\left(h(\eta)-\frac{f}{e^{5}} h\left(\frac{\eta-z_{1}}{e}\right)\right)|x-t|^{5} \\
& =\left(h(\eta)-h\left(\frac{\eta-z_{1}}{e}\right)+h\left(\frac{\eta-z_{1}}{e}\right)\left(1-\frac{f}{e^{5}}\right)\right)|x-t|^{5} \\
& \lesssim\left(\left|\eta-\frac{\eta-z_{1}}{e}\right|+\Delta\right)|x-t|^{5} \lesssim\left(|\eta(e-1)|+\left|z_{1}\right|+\Delta\right)|x-t|^{5} \\
& \lesssim \Delta\left(\frac{\delta}{u \Delta}\right)^{5 / 4} \lesssim \delta .
\end{aligned}
$$

Finally, we prove that $u$ can not be $\ll 1$. Assume that $u \ll 1$. Using the definition of $a_{4}$ and the estimates we obtained above, we obtain

$\left|a_{4}\right| \gtrsim\left|1-\frac{f}{e^{4}}\right|-\frac{f}{e^{4}}\left|1-w^{7}\right|-\left|t^{2}-\left(\frac{t-z_{1}}{e}\right)^{2}\right|-\left(\frac{t-z_{1}}{e}\right)^{2}\left|1-w^{7} \frac{f}{e^{4}}\right| \gtrsim \Delta$.

Hence, $u$ can not be $\ll 1$. This yields the upper bound for the arclength of the intersection.

Let $\min _{ \pm}(A \pm B)$ denote $\min (A+B, A-B)$.

Corollary 9 Let $d(z, 0)=\Delta \gtrsim \delta^{2 / 5}$. Then

i) The arclength of $E_{z}^{e, f} \cap \mathcal{N}\left(S^{1}, \delta\right)$ is $\lesssim\left(\frac{\delta}{\Delta}\right)^{1 / 4}$,

ii) if it is $\gtrsim\left(\frac{\delta}{u \Delta}\right)^{1 / 4}, 1 \lesssim u \ll(\Delta / \delta)^{1 / 3}$, then we have

$$
\min _{ \pm}\left(\left|(f e)^{2 / 3}-1 \pm d(z, 0)\right|\right) \lesssim \min \left((u \Delta)^{3 / 4} \delta^{1 / 4}, u^{3 / 2}(\delta / \Delta)^{1 / 2}\right)
$$


Proof. We divide $\mathcal{N}\left(S^{1}, \delta\right)$ into four segments; $\mathcal{N}\left(S^{1}, \delta\right)=\cup_{i=1}^{4} \mathcal{N}\left(S_{i}^{1}, \delta\right)$, where $\mathcal{N}\left(S_{1}^{1}, \delta\right)$ is as before and $\mathcal{N}\left(S_{i}^{1}, \delta\right)$ is obtained by rotating $\mathcal{N}\left(S_{1}^{1}, \delta\right)$ around the origin $i \pi / 2$ degrees. Note that if the intersection of the ellipse with $\mathcal{N}\left(S^{1}, \delta\right)$ is large, then its intersection with one of $\mathcal{N}\left(S_{i}^{1}, \delta\right)$ should be large, too.

Let $\left|E_{z}^{e, f} \cap \mathcal{N}\left(S_{1}^{1}, \delta\right)\right|>\left(\frac{\delta}{u \Delta}\right)^{1 / 4}$, for some $1 \lesssim u \ll(\Delta / \delta)^{1 / 3}$. Triangle inequality and (3.1) imply that

$$
\min _{ \pm}\left(\left|y_{1} \pm d(z, 0)\right|\right) \leq\left|z_{1}\right| \lesssim \min \left(u^{3 / 2}(\delta \Delta)^{1 / 2}, u^{9 / 4}(\delta / \Delta)^{3 / 4}\right)
$$

The fact that $e, f \in\left[\frac{1}{2}, 2\right]$ and (3.2) imply that

$$
\left|f-(e f)^{2 / 3}\right| \approx\left|f-e^{2}\right| \lesssim \min \left((u \Delta)^{3 / 4} \delta^{1 / 4}, u^{3 / 2}(\delta / \Delta)^{1 / 2}\right)
$$

Hence, we have

$$
f-1=(f e)^{2 / 3}-1+O\left(\min \left((u \Delta)^{3 / 4} \delta^{1 / 4}, u^{3 / 2}(\delta / \Delta)^{1 / 2}\right)\right)
$$

Using (3.23) and (3.24) in (3.3), we obtain

$$
\min _{ \pm}\left(\left|(f e)^{2 / 3}-1 \pm d(z, 0)\right|\right) \lesssim \min \left((u \Delta)^{3 / 4} \delta^{1 / 4}, u^{3 / 2}(\delta / \Delta)^{1 / 2}\right)
$$

Applying Theorem 8 (after a rotation) also in the cases where $\mathcal{N}\left(S_{1}^{1}, \delta\right)$ is replaced with $\mathcal{N}\left(S_{i}^{1}, \delta\right), i=2,3,4$ yields the claim of the corollary.

The following corollary proves Lemma 2. Let $E_{z}^{e, f, \theta}$ denote the ellipse $E_{z}^{e, f}$ rotated counter-clockwise by an angle $\theta$ around its center.

Corollary 10 Let $d(z, y)=\Delta \gtrsim \delta^{2 / 5}$. Then

i) The measure of the set $\mathcal{N}\left(E_{z}^{e, f, \theta}, \delta\right) \cap \mathcal{N}\left(E_{y}^{a, b}, \delta\right)$ is $\lesssim \delta\left(\frac{\delta}{\Delta}\right)^{1 / 4}$,

ii) if it is $\gtrsim \delta\left(\frac{\delta}{u \Delta}\right)^{1 / 4}, 1 \lesssim u \ll(\Delta / \delta)^{1 / 3}$, then we have

$$
\begin{aligned}
& \min _{ \pm}\left(\left|(f e)^{2 / 3}-(a b)^{2 / 3}\left(1 \pm d_{a, b}(z, y)\right)\right|\right) \lesssim \min \left((u \Delta)^{3 / 4} \delta^{1 / 4}, u^{3 / 2}(\delta / \Delta)^{1 / 2}\right) \\
& \text { where } d_{a, b}\left(\left(p_{1}, p_{2}\right),\left(q_{1}, q_{2}\right)\right)=\left(\left(p_{1}-q_{1}\right)^{2} / a^{2}+\left(q_{1}-q_{2}\right)^{2} / b^{2}\right)^{1 / 2}
\end{aligned}
$$

Proof. By a dilation, a translation and then a rotation, we can transform $E_{x_{0}, y_{0}}^{a, b}$ into $S^{1}$ and $E_{z}^{e, f, \theta}$ into $E_{w}^{e_{1}, f_{1}}$ such that $e_{1} f_{1}=\frac{e f}{a b}$ (since the area of $E_{z}^{e, f, \theta}$ is equal to the area of $E_{w}^{e_{1}, f_{1}}$ times $\left.a b\right)$ and $d(w, 0)=d_{a, b}(z, y)$. The claim follows by applying Corollary 9 to $E_{w}^{e_{1}, f_{1}}$ and $S^{1}$. 
Proof of Lemma 6: By making the suitable translations, rotations and dilations we can assume that $E_{2}=S^{1}$ and $E_{1}=E_{y}^{a, b}$, where $|y| \approx d$. We can further assume that $u \ll\left(\frac{\Delta}{\delta}\right)^{1 / 3}$, since the statement of the theorem is void if $u \gtrsim\left(\frac{\Delta}{\delta}\right)^{1 / 3}$.

Denote $u^{3 / 2}(\delta / \Delta)^{1 / 2}$ by $\xi$, and consider the functions

$$
\begin{aligned}
F(x) & =\left(|x|^{2}, d_{a, b}(x, y)^{2}\right), \\
G(r, s) & =\min _{ \pm}\left(\left|-1 \pm \sqrt{r}+(a b)^{2 / 3}(1 \pm \sqrt{s})\right|\right) .
\end{aligned}
$$

Theorem 10 implies that the set $S$ is contained in the set

$$
\bar{S}:=\left\{x \in \mathbb{R}^{2}:|x| \gtrsim \Delta, d(x, y) \gtrsim \Delta, G(F(x)) \lesssim \xi\right\} .
$$

It is easy to see that the measure of the set $B_{\xi}:=\{(r, s): G(r, s) \lesssim \xi\}$ is $\lesssim \xi$ (note that $\xi \lesssim 1$ ).

Below, we prove that the measure of the inverse image of a set of measure $\xi$ under $F$ is at most $(\xi / d)^{1 / 2}(|\log (\xi / d)|+1)$, which yields the claim of the lemma.

Let $B_{\xi}$ be a set of measure $\xi$ and $A_{\eta}$ be the set where the Jacobian of $F$, $J F$, is less then $\eta$. Co-area formula (see, e.g., [3] Theorem 3.2.3) implies that

$$
\left|F^{-1}\left(B_{\xi}\right)\right| \lesssim\left|A_{\eta}\right|+\frac{\xi}{\eta}
$$

Claim. $\left|A_{\eta}\right| \lesssim \frac{\eta}{d}\left|\log \left(\frac{\eta}{d}\right)\right|+1$.

Proof. Without loss of generality, we can assume that $\left|y_{1}\right| \gtrsim d$. It is easy to calculate that

$$
J F \approx \frac{x_{1}\left(x_{2}-y_{2}\right)}{b^{2}}-\frac{x_{2}\left(x_{1}-y_{1}\right)}{a^{2}}=x_{1} x_{2} \frac{a^{2}-b^{2}}{a^{2} b^{2}}-\frac{x_{1} y_{2}}{b^{2}}+\frac{x_{2} y_{1}}{a^{2}} .
$$

Hence,

$$
A_{\eta}=\left\{x \in \mathbb{R}^{2}: x_{1} \in(-2,2),\right.
$$

$$
\left.\left|x_{2}-\frac{x_{1} y_{2} a^{2}}{\left.x_{1}\left(a^{2}-b^{2}\right)+y_{1} b^{2}\right)}\right| \lesssim \frac{\eta a^{2} b^{2}}{\left|x_{1}\left(a^{2}-b^{2}\right)+y_{1} b^{2}\right|}\right\}
$$

This shows that if $\left|a^{2}-b^{2}\right| \ll d$, then $\left|A_{\eta}\right| \lesssim \frac{\eta}{d}$. Now, assume that $\left|a^{2}-b^{2}\right| \gtrsim d$. (3.27) implies that

$$
\left|A_{\eta}\right| \lesssim \int_{-2}^{2} \min \left(\frac{\eta a^{2} b^{2}}{\left|x_{1}\left(a^{2}-b^{2}\right)+y_{1} b^{2}\right|}, 1\right) \mathrm{d} x_{1} \lesssim \frac{\eta}{d}\left|\log \left(\frac{\eta}{d}\right)\right|+1
$$

which proves the claim.

Claim of the lemma follows from (3.26) and the claim above by choosing $\eta=(\xi d)^{1 / 2}$. 


\section{References}

[1] Bourgain, J.: Averages in the plane over convex curves and maximal operators. J. Analyse Math. 47 (1986), 69-85.

[2] CórdobA, A.: The Kakeya maximal function and spherical summation multipliers. Amer. J. Math. 99 (1977), 1-22.

[3] Federer, H.: Geometric measure theorey, Springer-Verlag Berlin Heideldberg, 1996.

[4] Kolasa, L., Wolff, T.: On some variants of the Kakeya problem. Pacific J. Math. 190 (1999), 111-154.

[5] Marstrand, J. M.: Packing circles in the plane. Proc. London Math. Soc. (3) $5 \mathbf{5}$ (1987), 37-58.

[6] Mitsis, T.: On a problem related to sphere and circle packing. J. London Math. Soc. (2) 60 (1999), 501-516.

[7] Mockenhaupt, G., Seeger, A., Sogge, C.: Wave front sets and Bourgain's circular maximal theorem. Ann. of Math. (2) 134 (1992), 207-218.

[8] Schlag, W.: A generalization of Bourgain's circular maximal theorem. J. Amer. Math. Soc. 10 (1997), 103-122.

[9] SchlaG, W.: A geometric inequality with applications to the Kakeya problem in three dimensions. Geom. Funct. Anal. 8 (1998), 606-625.

[10] Schlag, W.: A geometric proof of the circular maximal theorem. Duke Math. J. 93 (1998), 505-533.

[11] Wolff, T.: Recent work connected with the Kakeya problem. In Prospects in Mathematics (Princeton, N.J., 1996), ed. H. Rossi. American Mathematical Society, 1998.

Recibido: 25 de septiembre de 2001

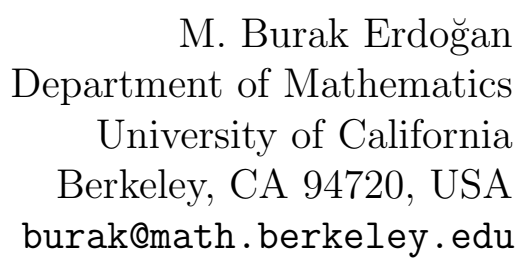

This result is a part of the author's Ph.D. thesis at the California Institute of Technology written under the guidance of Prof. Thomas Wolff. 